\title{
Managing tooth fractures
}

\begin{abstract}
Dr Sanjeev Bhanderi is a leading authority on the management of tooth fractures. At the British Dental Conference and Dentistry Show 2020 Dr Bhanderi will be sharing what he believes are the greatest challenges faced by clinicians in the area.
\end{abstract}

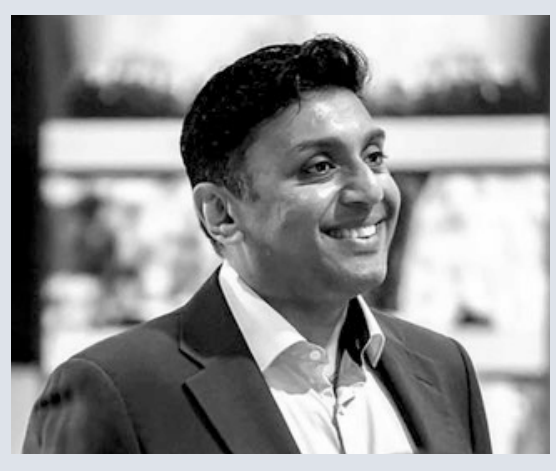

According to Dr Bhanderi: 'Fractures are becoming more and more prevalent in our patients for a number of reasons. First, people are keeping their teeth for much longer than they used to and therefore may be a long way down the 'restorative cycle' as they become more structurally compromised over time. Second, patients' expectations for any treatment or care they receive are higher. Third, as natural teeth are kept for longer, they are often associated with tooth wear. This might be compounded by the fact that more teeth will require endodontic treatment, which in itself can weaken teeth further.

'Another reason for the increased incidence of tooth fractures is that the longer teeth are kept and more heavily restored they become, the more likely they are to succumb to the longevity of occlusal load and forces.

'Finally, I believe that parafunction plays a significant role in the cause of tooth fracture, particularly in an increasingly younger cohort of patients. This might be due to psychological stress, odd biting habits, intraoral piercings, or in relation to larger restorations.

'The main challenge for clinicians is to identify the presence of a fracture early on. We can attempt to do this from patients' symptomology and by careful examination. The aim is to treat these teeth before they 'de-vitalise' or catastrophically split.'

Dr Bhanderi is widely renowned for his expertise within endodontics and is a registered Specialist in the field. He has worked as a Course Leader for the MSc in Endodontology at UCLan and is currently a Senior Lecturer at the University of Liverpool Dental School for the DDSc Endodontic Specialist training programme. He has lectured across the UK and internationally, and he is President Elect of the British Endodontic Society (BES).

Dr Bhanderi will be presenting in the Speciality Interest Theatre at the British Dental Conference and Dentistry show 2020, supported by the British Endodontic Society
(BES). His session will explore 'Tooth fractures: the beginning of the end?' and will give delegates plenty to think about.

He comments:

'During my session I will discuss the clinical signs and symptoms of fracture presentation, describing the protocols for and importance of clinical examination under magnification. I will also cover the relationship between fracture extent and prognosis, as well as treatment strategies for fractured teeth. The ultimate aim is always to prolong retention survival of compromised teeth for longevity and function.

'I hope that delegates will learn how to better assess fractures in teeth. They will leave with an improved understanding of how to manage a fractured tooth conservatively using the latest adhesive techniques. I also hope that professionals will increase their confidence in deciding between appropriate management of fractures or extraction, with valid patient consent obtained every time.'

The British Dental Conference and Dentistry Show 2020 will be held on Friday 15 and Saturday 16 May at the Birmingham NEC, co-located with DTS.

www.thedentistryshow.co.uk

\section{Face mask stockpile uncovered}

The British Dental Association (BDA) has applauded swift action from government and the dental industry to unlock stocks of face masks to ease the mounting supply problems facing UK dental services.

Dentist leaders anticipated some larger practices having to scale down activity or prioritise urgent over routine care in

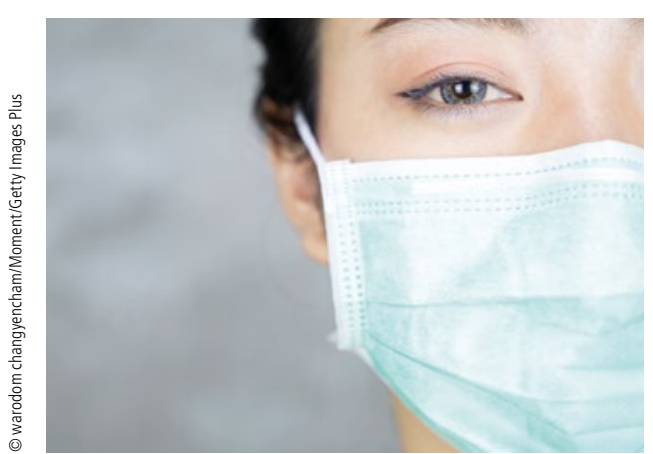

February. Shortages have emerged in the wake of the coronavirus (COVID-19) outbreak, following panic buying, and moves by China - the world's leading supplier of hygienic masks - to prioritise unprecedented domestic demand over the export market.

Following BDA concerns, the Department of Health and Social Care has indicated significant central stockpiles existed, including reserves built up for the UK's departure from the European Union.

These stocks formed part of the supply chain for hospitals but were out of reach for primary care providers like dentists and GPs who access products directly from manufacturers and wholesalers.

In an agreement forged in recent days the $\mathrm{BDA}$ understands supplies from government stockpiles are now being released to ease immediate pressures.

The face mask shortage has gone global, with dentists in Japan, Australia, New Zealand, Canada and parts of the US reporting major disruptions in supply.

British Dental Association Chair Mick Armstrong: 'The clock was ticking on this face mask shortage, and dentists and patients will applaud decisive action from the industry and government.

'We were clear any solution hinged on getting needed supplies into the hands of high street practices. That process kicks off today, and not a moment too soon.

'Our members are facing difficult choices, and we will continue doing everything in our power to prevent disruption to patient care.' 